\title{
EFFECTS OF HIGH FLAVANOL DARK CHOCOLATE ON CARDIOVASCULAR FUNCTION AND PLATELET AGGREGATION
}

Gurvinder Rull, Zetty N. Mohd-Zain, Julian Shiel, Martina H. Lundberg, David J. Collier, Atholl Johnston, Timothy D. Warner and Roger Corder.

William Harvey Research Institute, Barts and the London School of Medicine and Dentistry, Queen Mary University of London, Charterhouse Square, LONDON, EC1M 6BQ, UK

\section{Corresponding author:}

Prof. Roger Corder, William Harvey Research Institute, Charterhouse Square, London, EC1M 6BQ, UK. E-mail: r.corder@qmul.ac.uk Tel: +44 2078828109. 


\section{Graphical Abstract}
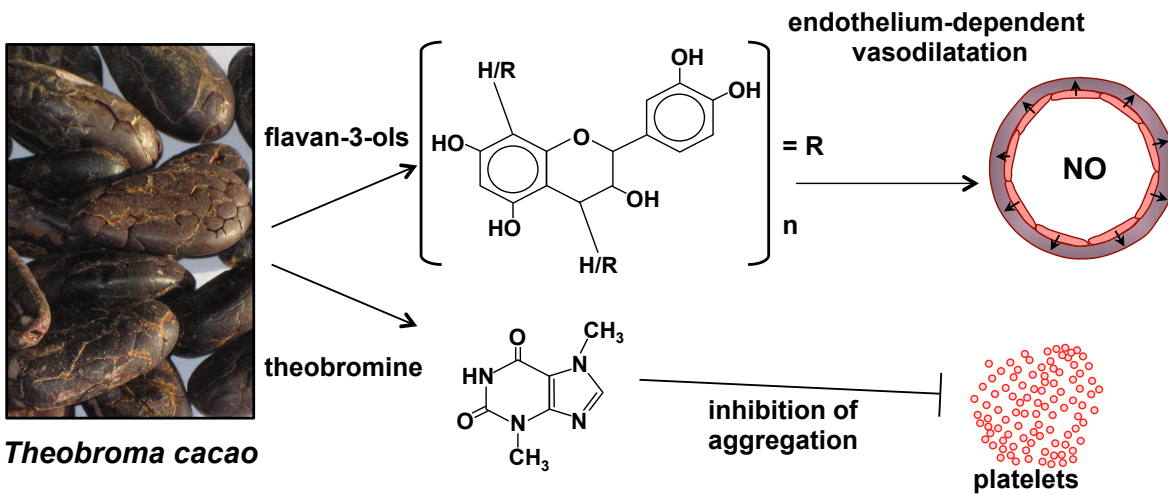


\begin{abstract}
Regular consumption of chocolate and cocoa products has been linked to reduced cardiovascular mortality. This study compared the effects of high flavanol dark chocolate (HFDC; 1064mg flavanols/day for 6 weeks) and low flavanol dark chocolate (LFDC; 88mg flavanols/day for 6 weeks) on blood pressure, heart rate, vascular function and platelet aggregation in men with pre-hypertension or mild hypertension. Vascular function was assessed by pulse wave analysis using radial artery applanation tonometry in combination with inhaled salbutamol $(0.4 \mathrm{mg})$ to assess changes due to endothelium-dependent vasodilatation. HFDC did not significantly reduce blood pressure compared to baseline or LFDC. Heart rate was increased by LFDC compared to baseline, but not by HFDC. Vascular responses to salbutamol tended to be greater after HFDC. Platelet aggregation induced by collagen or the thromboxane analogue U46619 was unchanged after LFDC or HFDC, whereas both chocolates reduced responses to ADP and the thrombin receptor activator peptide, SFLLRNamide (TRAP6), relative to baseline. Pre-incubation of platelets with theobromine also attenuated platelet aggregation induced by ADP or TRAP6. We conclude that consumption of HFDC confers modest improvements in cardiovascular function. Platelet aggregation is modulated by a flavanol-independent mechanism that is likely due to theobromine.
\end{abstract}

Keywords: platelet, PDE inhibitor, blood pressure, heart rate, pulse pressure amplification ratio

\title{
Chemical compounds studied in this article:
}

theobromine (PubChem CID 5429); prostacyclin (PubChem CID 5280427) 


\section{Introduction}

Regular consumption of dark chocolate has been linked to a lower incidence of myocardial infarction and stroke, and to an overall reduction in cardiovascular mortality [1-4]. Such observations are consistent with reports that cocoa products lower blood pressure [5-8], improve endothelium-dependent vasodilator responses [7-13], and inhibit platelet aggregation [12-18].

The actions of cocoa and dark chocolate have been attributed primarily to the high level of flavan-3-ols. However, the flavanol content of cocoa products is highly variable depending on post-harvest treatment of cocoa beans. Fermentation, drying and roasting reduce total flavanol content, with the result that commercial dark chocolate typically contain $<1 \%$ flavanols by weight $[19,20]$. Cocoa flavanols are composed of monomers, primarily (-)-epicatechin (10 $40 \%$ total flavanols), and procyanidins, which are short oligomers composed of epicatechin and catechin units, mainly dimer to decamer $(20-80 \%$ total flavanols) $[19,20]$. Cocoa products also contain high levels of methylxanthines, theobromine $(0.5-2.0 \%)$ and caffeine $(0.03-0.2 \%)[20]$, which may act as phosphodiesterase inhibitors or adenosine receptor antagonists [21-23]. The specific contributions of flavanols and methylxanthines components to the cardioprotective effects of regular cocoa or dark chocolate consumption have yet to be fully defined.

Patient and volunteer studies of the effects of dark chocolate and cocoa on blood pressure, endothelial function, and platelet aggregation, have often lacked adequate blinding because inappropriate placebo products (such as white chocolate) were used, which lack flavanols and theobromine $[5-7,9,12,14-18,24]$. Here to investigate the role of flavanols in mediating the actions of dark chocolate on blood pressure and platelet function we compared responses to a high flavanol dark chocolate (HFDC) with a placebo low flavanol dark chocolate (LFDC) in a double-blind crossover study in men with pre-hypertension or mild hypertension. 


\section{Methods}

\subsection{Study Design and Participants}

The study was a single centre randomized double-blind placebo controlled investigation with a crossover design. Ethical approval for the study was obtained from the NHS Research Ethics Service (East London and the City Research Ethics Committee; REC reference number: 07/Q0605/44). Volunteer participants were recruited through the east London GP network using a database search for men with recently diagnosed untreated mild hypertension (SBP 140 - 159 mmHg; DBP 90 - $99 \mathrm{mmHg}$ ) or prehypertension (SBP 120 - $139 \mathrm{mmHg}$; DBP $80-89 \mathrm{mmHg}$ ). Potential participants were sent a letter describing the study, which invited them to be screened for eligibility. Pre-recruitment screening involved clinic measurement of blood pressure, and $24 \mathrm{~h}$ ambulatory blood pressure measurement (ABPM) to verify that daytime blood pressure was consistent with prehypertension or mild hypertension. Additional criteria were aged $>45$ years and $<70$ years, otherwise healthy and free of other disease (confirmed by physical examination, 12-lead ECG, and routine blood chemistry including liver function tests).

Specific exclusion criteria were: diabetes mellitus or raised fasting blood glucose; serum cholesterol $>7 \mathrm{mmol} / \mathrm{L}$; BMI $>30 \mathrm{~kg} / \mathrm{m}^{2}$; history of cardiac arrhythmias or ECG abnormalities at baseline; history of psychiatric or psychological illness; history of hypo- or hyperthyroidism; participation in another trial whether active or in follow-up; abnormal liver function tests or other routine blood chemistry; excessive alcohol consumption; regular medicines for any condition; regular use of herbal medicines or other alternative remedies. For those recruited to the study the following additional requirements were set: no red wine consumption during the study; no aspirin or other non-steroidal anti-inflammatory agents for 4 weeks before the study or during the study (allowable pain relief paracetamol); use of any medicines during the study to be recorded. Men meeting the recruitment criteria and electing 
to participate in the study were randomized to consume HFDC (50 g/day; $25 \mathrm{~g}$ each morning and $25 \mathrm{~g}$ in the afternoon) or LFDC (50 g/day) of similar taste and appearance for 6 weeks. At the end of this period participants were crossed over to receive the other chocolate for 6 weeks.

A cohort size of 32 participants was chosen based on based on previous reports of blood pressure decreases of $5 \mathrm{mmHg}$ after dark chocolate consumption [7]. With a crossover design this gave $97 \%$ power to measure a difference of $5 \mathrm{mmHg}$ with a significance of 0.05 after HFDC compared to LFDC, and $80 \%$ power to detect a change in any variable by more than 0.5 of the standard deviation of its measurement.

\subsection{Dark Chocolate Products and Randomization}

HFDC and LFDC were from Barry Callebaut (Lebbeke-Wieze, Belgium). The compositions of the two chocolates are shown in table 1. The randomisation schedule was sent as a password-protected file to Barry Callebaut, who prepared separate patient coded boxes for each phase of the study (50 g bars x 42 per box, plain foil wrapped). The investigators were blinded to the randomisation schedule. Flavanol doses for LFDC (88 mg/day) and HFDC (1064 mg/day) were chosen based on published observations that $\approx 80 \mathrm{mg}$ total flavanols/day was without significant effect on endothelial function in previous studies, whereas large doses of $\approx 900 \mathrm{mg} /$ day produce a consistent and marked increase in endothelial function [10].

\subsection{Study measurements}

The main study measurements were made at baseline, and at 6 and 12 weeks - after completion of each phase of dark chocolate consumption. Blood samples were collected for full lipid profile at baseline, 3, 6, 9 and 12 weeks. Three-week intervals were used to ensure that any changes in plasma lipid profiles reached steady state [25]. Platelet aggregation responses were assessed at baseline, 6 and 12 weeks. Blood pressure and heart rate were recorded as the mean of three measurements using an Omron BP Monitor 705CP II at 
baseline, 3, 6, 9 and 12 weeks. At 6 and 12 weeks pulse wave analysis (PWA) was undertaken by radial artery tonometry using a Millar tonometer in conjunction with SphygmoCor Px Model software analysis to derive haemodynamic indices. Pulse pressure amplification ratio (PPA) was expressed as the ratio of central pulse pressure (CPP) to peripheral (brachial) pulse pressure (PPP). Changes in vascular function as a result of endothelium-dependent vasodilatation were assessed by PWA at 10 and 15 min after inhalation of salbutamol (0.4 $\mathrm{mg}$ ) [26]. ABPM recordings (24 h) were made during participant screening and at 6 and 12 weeks using a Spacelabs ABP monitor 90207 (Dolby UK, Stirling). Twenty-one subjects had ABPM recordings at both 6 and 12 weeks (reasons for missing recordings: failure to attend the clinic on the required day, or BP monitor recording failure at either 6 or 12 weeks). Caffeine and theobromine were measured by HPLC following published methodology [27]. Plasma lipids (total cholesterol, LDL-cholesterol, HDL-cholesterol and triglycerides) were measured by the Pathology Laboratories at Barts Health NHS trust.

\subsection{Platelet Function}

Platelet aggregation with simultaneous measurement of responses to a range of agonist concentrations was performed as previously described using a 96-well plate methodology [28]. Blood was collected by venepuncture into tri-sodium citrate $(3.2 \%, 1: 9 \mathrm{v} / \mathrm{v}$; Sigma, Poole, Dorset, U.K.) and centrifuged (175 x g, $15 \mathrm{~min})$ to obtain platelet-rich plasma (PRP). At baseline, 6 and 12 weeks, platelet aggregation responses were assessed by transferring samples of PRP (100 $\mu \mathrm{L} /$ well) into a 96-well plate (Nunc, Lutterworth, Leicestershire, U.K.) containing $10 \mu \mathrm{L}$ of vehicle or agonist: adenosine diphosphate (ADP; $0.1-30 \mu \mathrm{M}$; LabMedics, Salford, Manchester, U.K.), collagen (0.1-30 $\mu \mathrm{g} / \mathrm{ml}$; Nycomed, Linz, Austria), the PAR-1 activator peptide TRAP6 (SFLLRNamide; 0.1 - $30 \mu \mathrm{M}$; Bachem, Bubendorf, Switzerland), or the stable TXA2-mimetic U46619 (0.1 - $30 \mu \mathrm{M}$; Cayman Chemical Company, Ann Arbor, MI, U.S.A.). The plate was placed into a 96-well plate reader (Tecan 
Sunrise) at $37^{\circ} \mathrm{C}$, and absorbance was measured at $595 \mathrm{~nm}$ every $15 \mathrm{~s}$ for 16 minutes with vigorous shaking between readings. Percentage aggregation was calculated with reference to the absorbance of platelet-poor plasma (obtained by centrifugation of PRP at 15,000 $\mathrm{g}$ for 2 $\min$ ) as a surrogate for $100 \%$ aggregation. For studies of the effect of theobromine blood samples were collected from healthy volunteers. PRP was pre-incubated for 30 min with theobromine (Sigma, Poole, Dorset, U.K.) at the concentrations indicated. All graphs show aggregation responses at $10 \mathrm{~min}$.

\subsection{Statistical Analyses}

Twenty-six subjects completed both phases of the 12-week study. Data were analyzed by using MiniTab or GraphPad Prism (GraphPad Software Inc, CA, USA) software. Results are reported as mean $\pm \mathrm{SEM}$. Statistical differences for mean data obtained from PWA were analysed by two way ANOVA using MiniTab General Linear Model. Statistical analyses for platelet studies were conducted using GraphPad Prism. Concentration-response curves were compared by two way ANOVA with Bonferroni post-hoc tests.

\section{Results}

\subsection{Blood pressure and heart rate changes}

The study population was generally middle aged, and mainly pre-hypertensive based on daytime ambulatory blood pressure measurements (Table 2). No significant changes in systolic or diastolic blood pressure were observed after HFDC or LFDC, whether measured by $24 \mathrm{~h}$ ambulatory BP recording or in the clinic. There was a tendency for lower SBP and MAP $(\approx-2 \mathrm{mmHg})$ after HFDC for both overall and daytime ambulatory BP measurements, but this did not reach significance. Heart rate was increased after LFDC by $\approx 4$ bpm compared to pre-study values. After HFDC it was not different from baseline and significantly less than after LFDC (Tables 3 and 4). 


\subsection{Pulse wave analyses and response to salbutamol}

Basal measurements of peripheral and central SBP, DBP and PP showed no significant differences after HFDC compared to LFDC (Table 4 and Figure 1). Augmentation pressure (AP) and augmentation index (AI) were also not significantly different. Heart rate, difference between peripheral and central SBP (PSBP - CSBP), and PPA were all significantly lower after HFDC compared to values after LFDC (Table 4 and Figure 1). Salbutamol causes endothelium-dependent vasodilatation [26]. Consistent with this, peripheral DBP, and central SBP and DBP decreased significantly after salbutamol (Figure 1). Salbutamol tended to have greater effects on blood pressure measurements after HFDC but these changes did not reach significance (Figures 1 and 2). AP and AI were reduced by salbutamol but HFDC did not significantly modify these responses. Basal PSBP - CSBP and PPA were lower after HFDC compared to LFDC (Table 4). Salbutamol increased PSBP - CSBP and PPA with a marginally significant difference remaining after HFDC compared to LFDC (Figure 1). For PPA the average increases after salbutamol from basal (expressed as $\%$ change) were greater after HFDC (10 min and 15 min; HFDC: $6.06 \pm 1.22$ and $6.51 \pm 1.20$; LFDC: $3.81 \pm 1.11$ and $4.10 \pm 1.20 ; \mathrm{P}=0.005$ ) (Figure 2). Heart rate was unaffected by salbutamol and remained significantly lower after HFDC (Figure 1).

\subsection{Platelet aggregation}

Platelet aggregation responses after consuming HFDC for 6 weeks were not significantly different from those recorded after LFDC (Figure 3). However, while neither HFDC nor LFDC had any effect on the responses to collagen or U46619 compared to baseline (Figure 3a and 3b), both HFDC and LFDC suppressed significantly platelet aggregation induced by ADP $(\mathrm{P}=0.01)$ and TRAP6 $(\mathrm{P}=<0.001)$ relative to baseline responses (Figure $3 \mathrm{c}$ and $3 \mathrm{~d})$. Based on the areas under the curve for 3, 10 and $30 \mu \mathrm{M}$ agonist concentrations, HFDC and LFDC reduced ADP responses by $\approx 30 \%$ and TRAP 6 responses by $\approx 60 \%$. 
Both chocolates contained similar levels of theobromine and caffeine (Table 1), which can act as phosphodiesterase (PDE) inhibitors [21], a well-established mechanism for inhibiting platelet aggregation [29]. Theobromine plasma levels were comparable after HFDC and LFDC, and significantly greater than baseline (Table 5). To test whether the inhibitory effect of HFDC and LFDC on ADP and TRAP6 responses was due to actions of methylxanthines PRP was pre-incubated with theobromine before incubation with ADP and TRAP6. Theobromine $(2-50 \mu \mathrm{g} / \mathrm{ml})$ significantly reduced platelet aggregation observed with ADP (P $=<0.001$ ) but not with TRAP6 (Figure 4a and 4b). To test whether the anti-aggregatory effects of theobromine were mediated via cAMP the inhibitory effect of prostacyclin was assessed in the presence and absence of theobromine. Theobromine caused a significant leftward shift of the prostacyclin curve using ADP as the agonist for stimulating platelet aggregation $(\mathrm{P}=0.028)$, representing an approximately 2 -fold increase in the sensitivity to prostacyclin (Figure 4c). Similar results were observed using TRAP6 as the stimulus for platelet aggregation $(\mathrm{P}=0.005)$ (Figure $4 \mathrm{~d})$.

\subsection{Serum Lipids}

No changes were observed in total cholesterol, LDL-cholesterol, HDL-cholesterol or triglycerides after HFDC or LFDC (Table 5).

\section{Discussion}

\subsection{Overview}

The age-related increased risk of an atherothrombotic event - myocardial infarction or stroke - is generally viewed as a complex relationship evolving over many years. It is frequently linked to deterioration in vascular function, increased prevalence of proinflammatory changes, raised blood pressure, dyslipidaemia, and platelet thrombogenicity. These are all changes that cocoa flavanols may modulate [1-18]. Here, HFDC tended to lower SBP and 
improved vascular function, compared to LFDC, but the effects generally failed to reach significance. Increased flavanol intake reduced heart rate relative to LFDC - an effect that could potentially modify cardiovascular risk. Both HFDC and LFDC suppressed platelet aggregation, probably as a result of theobromine acting as a PDE inhibitor, rather than through an effect of flavanols.

\subsection{Effects of flavanols on blood pressure and heart rate}

A number of studies have investigated the effect of dark chocolate or cocoa on blood pressure. A meta-analysis of 20 studies of mainly healthy volunteers, reported a mean reduction in SBP of $-2.77 \mathrm{mmHg}(95 \% \mathrm{CI}-4.72,-0.82 ; \mathrm{P}=0.005)$, and $\mathrm{DBP}$ of $-2.20 \mathrm{mmHg}$ (95\% CI $-3.46,-0.93 ; \mathrm{P}=0.006)$ [24]. The effect was greater for trials that compared active product to a flavanol free control product $(\mathrm{SBP}-3.70 \mathrm{mmHg}, 95 \% \mathrm{CI}-6.02,-1.36, \mathrm{P}=0.002$; DBP $-2.71 \mathrm{mmHg}, 95 \% \mathrm{CI}-4.26,-1.50, \mathrm{P}=<0.001)$ [24]. But most investigations using flavanol free placebo products were poorly controlled, and not double-blinded. In ten studies where blinding was achieved by matching a low flavanol control product to the active high flavanol product, the meta-analysis showed no significant changes in blood pressure (SBP $0.71 \mathrm{mmHg}, 95 \% \mathrm{CI}-2.99,1.57$; DBP $-0.78 \mathrm{mmHg}, 95 \% \mathrm{CI}-2.26,0.70)$ [24]. This is consistent with results described here where a trend for SBP reduction of $\approx 2 \mathrm{mmHg}$ was observed with HFDC compared to LFDC, and DBP was unchanged. A reduction in SBP of 2 $\mathrm{mmHg}$ would be medically important if sustained over time and reproducible across a larger population as this could equate to a reduction in stroke risk of $\approx 10 \%[30]$.

Compared with baseline values, LFDC significantly increased heart rate. This is likely an effect of methylxanthines (theobromine and caffeine), which cause sympathetic activation primarily through adenosine receptor blockade $[22,23]$. Heart rate was not increased with HFDC even though it had similar methylxanthine content, indicating that flavanols modulate sympathetic activation. In agreement with this, treatment for six months with a diet enriched 
in cocoa flavanols resulted in $\approx 25 \%$ lower heart rate in a strain of rabbits, which are genetically prone to hypercholesterolaemia, compared to age-matched control rabbits of the same strain [31]. This difference in heart rate was attributed to cocoa flavanols preventing the decline in parasympathetic function observed in control hypercholesterolaemic animals [31]. Here, a flavanol-dependent increase in parasympathetic tone, suppressing methylxanthineinduced sympathetic activation, may explain the lower heart rate after HFDC compared with LFDC.

Elevated resting heart rate, with increased sympathetic activation, reduced vagal tone, and delayed heart rate recovery after exercise are associated with increased mortality particularly in patients with pre-existing CVD $[32,33]$. Hence, flavanol-induced improvement in sympathovagal balance may be a previously unrecognised beneficial effect associated with consumption of flavanol-rich cocoa products.

\subsection{Pulse wave analysis}

The difference between central and peripheral PP plays a critical role in cardiovascular risk $[34,35]$. Previous investigations of the effects of antihypertensive drugs on parameters derived from PWA indicate that reduced CSBP and CPP are associated with improved outcome [36]. Reduced risk after anti-hypertensive therapy is associated with increased PPA, mainly as a result of the vasodilator effects of antihypertensive agents. Here PPA was decreased, which might be considered detrimental. This was likely due to the reduction in heart rate as it leads to delayed timing of the reflected wave, which then merges with PP and increases AP [34]. Consistent with this PPA was inversely correlated with AP $\left(\mathrm{r}^{2}=0.78\right.$, data not shown). Summation of the reflected wave with central PP is thought to adversely affect cardiac function by prolonging ejection duration and increasing afterload [34]. However, the decrease in PPA after HFDC may be an anomalous observation. Athletes also have lower HR and reduced PPA, which is attributed to peripheral vasodilatation, reduced wave reflection and 
lower pulse wave velocity $[37,38]$. Indeed, previous studies have reported a reduction in pulse wave velocity after dark chocolate $[39,40]$, which in combination with lower heart rate could have contributed to the reduction in PPA after HFDC.

After HFDC salbutamol tended to have a greater effect on most parameters measured, but only PPA increased significantly more (Figure 2, $\mathrm{P}=0.005$ ). Nevertheless this indicates greater salbutamol-induced vasodilatation after HFDC. Based on previous studies and results described here, flavanols have only a modest effect on blood pressure, despite improving endothelium-dependent vasodilator responses (flow-mediated dilatation or salbutamol) [713], and reducing vascular stiffness (assessed by pulse wave velocity) $[39,40]$. These actions may have particular relevance for preventing cardiovascular aging, as reduced endotheliumdependent vasodilatation is associated with increased risk of hypertension in elderly subjects $[41,42]$. The degree to which cocoa flavanols increase vascular compliance and decrease cardiovascular disease risk, particularly in relation to increasing age, requires long-term studies in larger cohorts to assess whether such effects are sustained or develop further over time.

\subsection{Platelet aggregation}

ADP, collagen, the PAR-1 activator peptide TRAP6, and the stable TXA 2 -mimetic U46619 were used to stimulate platelet aggregation as these agonists are widely used for in vitro platelet testing [28]. Hypertension is frequently associated with a prothrombotic state with increased platelet thrombogenecity [43]. Previous studies of platelet aggregation after consumption of high flavanol chocolate and cocoa have ascribed anti-platelet effects to flavanols [13-18]. However, many of these studies were poorly controlled using low flavanol placebo products devoid of theobromine [14-18].

Here, platelet aggregation induced by thrombin-receptor activation or ADP was attenuated to a similar degree after consumption of LFDC or HFDC, compared to baseline responses. 
Stimulation of platelets with ADP or thrombin inhibits adenylate cyclase activity and increases phosphodiesterase (PDE) activity in platelets, allowing these platelet agonists to override the inhibitory effect of cAMP [44]. Hence PDE inhibitors, including methylxanthines, reduce ADP and PAR1 responses and are potent suppressors of platelet aggregation [29]. As HFDC and LFDC contained comparable levels of theobromine, it seemed likely that PDE inhibition accounted for the decrease in platelet responses. However, addition of theobromine to platelet-rich plasma caused only modest reductions in ADP and TRAP6 responses, which only reached significance with ADP. Nevertheless, it augmented the effect of prostacyclin, confirming that theobromine promotes cAMP-dependent decreases in platelet aggregation via PDE inhibition. The inhibitory profile of theobromine is very similar to the nonselective PDE inhibitor pentoxifylline and the selective PDE3 inhibitor cilostazol, which have limited effect alone, yet show markedly increased inhibition of platelet aggregation in the presence of prostacyclin [29].

A number of other factors may have reduced the effectiveness of theobromine when added directly to PRP, compared to the observed reductions in ADP and TRAP6 responses after LFDC or HFDC consumption for 6 weeks. Theobromine has poor lipid solubility so that 30 min pre-incubation may be insufficient for transmembrane equilibrium to be reached. Maximum PDE inhibition may require daily consumption of high levels of theobromine in order to reach high steady state levels in platelets, with high levels in portal blood after gastrointestinal absorption leading to greater changes in platelet function. Theobromine may also confer a greater anti-aggregatory action in the circulation, and in collected blood samples, by increasing and then preserving platelet cAMP and cGMP generated through exposure to endothelial prostacyclin and nitric oxide. This phenomenon has been observed with sildenafil, which has no effect when added directly to PRP, but significantly inhibits ex vivo platelet aggregation after oral dosing [29]. 
Based on results described here, evidence that chocolate consumption is associated with decreased risk of stroke may well be as much due to theobromine as to the presence of flavanols, since milk chocolate consumption is also associated with reduced cardiovascular mortality [4]. Indeed, PDE inhibition with theobromine may well be sufficient to reduce atherothrombotic events as the selective PDE3 inhibitor cilostazol reduces stroke risk [29]. Studies of chocolate consumption indicate regular daily intake of $\approx 10 \mathrm{~g} /$ day is sufficient to confer a benefit [1-4]. HFDC and LFDC both contained $\approx 0.8 \%$ theobromine, which is comparable to other dark chocolates $(0.5-1.6 \%)$ [20]. Milk chocolates also contain theobromine $(0.1-0.2 \%)$ [20]. But the threshold daily amount of theobromine to modulate platelet function requires further investigation. Studies of coffee consumption, where caffeine is clearly a key bioactive, have also shown a reduced risk of CHD mortality and ischaemic stroke in habitual coffee drinkers [45-47]. When these findings are considered in conjunction with observations from chocolate studies, this suggests that daily intake of methylxanthines is a key dietary factor modulating platelet function.

\subsection{Changes in plasma lipid profile}

Cocoa fat is composed of approximately $33 \%$ stearic acid, $25 \%$ palmitic acid, and $33 \%$ monounsaturated fat, and has a largely neutral effect on cholesterol levels [48]. In agreement with this, total cholesterol, HDL-cholesterol, LDL-cholesterol and triglycerides were unchanged during 12 weeks consumption of HFDC and LFDC.

\subsection{Limitations of the study}

The study was underpowered for confirming blood pressure lowering effects of flavanols. Measurement of pulse wave velocity as an index of vascular stiffness would have complemented PWA measurements after HFDC and LFDC, and following salbutamol challenge. Comparison of flow-mediated vasodilatation with responses to salbutamol would have provided additional insight into the changes in endothelial function after HFDC. The 
effects of theobromine on heart rate, blood pressure, and platelet function are important confounders when attempting to discriminate the effects of the flavanol constituents of dark chocolate.

\section{Conclusions}

Clinical trials of blood pressure lowering drugs show consistent reductions in the incidence of stroke [49]. However, there is still scope for better treatment of hypertension, particularly in the elderly with systolic hypertension where endothelial dysfunction is an unmet clinical need [41,50]. In addition, exaggerated blood pressure responses during exercise with reduced heart rate recovery post exercise are associated with increased cardiovascular mortality, due to endothelial dysfunction and sympathetic activation [32,33]. Hence, new insights into the extent flavanols modify cardiovascular risk may be obtained by investigating effects on responses to exercise.

Acknowledgements Mr. Clovell M. David for technical assistance and Mr. Michael G. Taylor for patient recruitment.

Funding This study was supported by a grant (to R. Corder) from Barry Callebaut Belgium NV.

\section{References}

[1] Buijsse B, Feskens EJ, Kok FJ, Kromhout D. Cocoa intake, blood pressure, and cardiovascular mortality: the Zutphen Elderly Study. Arch Intern Med 2006;166:411-7.

[2] Buijsse B, Weikert C, Drogan D, Bergmann M, Boeing H. Chocolate consumption in relation to blood pressure and risk of cardiovascular disease in German adults. Eur Heart $\mathbf{J}$ 2010;31:1616-23. 
[3] Larsson SC, Virtamo J, Wolk A. Chocolate consumption and risk of stroke: a prospective cohort of men and meta-analysis. Neurology 2012;79:1223-9.

[4] Janszky I, Mukamal KJ, Ljung R, Ahnve S, Ahlbom A, Hallqvist J. Chocolate consumption and mortality following a first acute myocardial infarction: the Stockholm Heart Epidemiology Program. J Intern Med 2009;266:248-57.

[5] Grassi D, Lippi C, Necozione S, Desideri G, Ferri C. Short-term administration of dark chocolate is followed by a significant increase in insulin sensitivity and a decrease in blood pressure in healthy persons. Am J Clin Nutr 2005;81:611-4.

[6] Taubert D, Berkels R, Roesen R, Klaus W. Chocolate and blood pressure in elderly individuals with isolated systolic hypertension. JAMA 2003;290:1029-30.

[7] Grassi D, Necozione S, Lippi C, Croce G, Valeri L, Pasqualetti P, et al. Cocoa reduces blood pressure and insulin resistance and improves endothelium-dependent vasodilation in hypertensives. Hypertension 2005;46:398-405.

[8] Engler MB, Engler MM, Chen CY, Malloy MJ, Browne A, Chiu EY, et al. Flavonoid-rich dark chocolate improves endothelial function and increases plasma epicatechin concentrations in healthy adults. J Am Coll Nutr 2004;23:197-204.

[9] Heiss C, Jahn S, Taylor M, Real WM, Angeli FS, Wong ML, et al. Improvement of endothelial function with dietary flavanols is associated with mobilization of circulating angiogenic cells in patients with coronary artery disease. J Am Coll Cardiol 2010;56:218-24. [10] Schroeter H, Heiss C, Balzer J, Kleinbongard P, Keen CL, Hollenberg NK, et al. (-)Epicatechin mediates beneficial effects of flavanol-rich cocoa on vascular function in humans. Proc Natl Acad Sci USA 2006; 103:1024-9.

[11] Balzer J, Rassaf T, Heiss C, Kleinbongard P, Lauer T, Merx M, et al. Sustained benefits in vascular function through flavanol-containing cocoa in medicated diabetic patients a double-masked, randomized, controlled trial. J Am Coll Cardiol 2008;51:2141-9. 
[12] Hermann F, Spieker LE, Ruschitzka F, Sudano I, Hermann M, Binggeli C, et al. Dark chocolate improves endothelial and platelet function. Heart 2006;92:119-20.

[13] Flammer AJ, Sudano I, Wolfrum M, Thomas R, Enseleit F, Périat D, et al.

Cardiovascular effects of flavanol-rich chocolate in patients with heart failure. Eur Heart J 2012;33:2172-80.

[14] Rein D, Paglieroni TG, Wun T, Pearson DA, Schmitz HH, Gosselin R, et al. Cocoa inhibits platelet activation and function. Am J Clin Nutr 2000;72:30-5.

[15] Holt RR, Schramm DD, Keen CL, Lazarus SA, Schmitz HH. Chocolate consumption and platelet function. JAMA 2002;287:2212-3.

[16] Murphy KJ, Chronopoulos AK, Singh I, Francis MA, Moriarty H, Pike MJ, et al. Dietary flavanols and procyanidin oligomers from cocoa (Theobroma cacao) inhibit platelet function. Am J Clin Nutr 2003;77:1466-73.

[17] Innes AJ, Kennedy G, McLaren M, Bancroft AJ, Belch JJ. Dark chocolate inhibits platelet aggregation in healthy volunteers. Platelets 2003;14:325-7.

[18] Flammer AJ, Hermann F, Sudano I, Spieker L, Hermann M, Cooper KA, et al. Dark chocolate improves coronary vasomotion and reduces platelet reactivity. Circulation 2007; 116:2376-82.

[19] Gu L, House SE, Wu X, Ou B, Prior RL. Procyanidin and catechin contents and antioxidant capacity of cocoa and chocolate products. J Agric Food Chem 2006;54:4057-61. [20] Langer S, Marshall LJ, Day AJ, Morgan MR. Flavanols and methylxanthines in commercially available dark chocolate: a study of the correlation with nonfat cocoa solids. J Agric Food Chem 2011;59:8435-41.

[21] Butcher RW, Sutherland EW. Adenosine 3',5'-phosphate in biological materials. I. Purification and properties of cyclic 3',5'-nucleotide phosphodiesterase and use of this enzyme to characterize adenosine 3',5'-phosphate in human urine. J Biol Chem 1962;237:1244-50. 
[22] Fredholm BB, Bättig K, Holmén J, Nehlig A, Zvartau EE. Actions of caffeine in the brain with special reference to factors that contribute to its widespread use. Pharmacol Rev $1999 ; 51: 83-133$

[23] Yu L, Coelho JE, Zhang X, Fu Y, Tillman A, Karaoz U, et al. Uncovering multiple molecular targets for caffeine using a drug target validation strategy combining A 2 A receptor knockout mice with microarray profiling. Physiol Genomics 2009;37:199-210.

[24] Ried K, Sullivan TR, Fakler P, Frank OR, Stocks NP. Effect of cocoa on blood pressure. Cochrane Database Syst Rev. 2012;8:CD008893.

[25] Kris-Etherton PM, Dietschy J. Design criteria for studies examining individual fatty acid effects on cardiovascular disease risk factors: human and animal studies. Am J Clin Nutr 1997;65(5 Suppl):1590S-6S.

[26] Rambaran C, Jiang B, Ritter JM, Shah A, Kalra L, Chowienczyk PJ. Assessment of endothelial function: comparison of the pulse wave response to $ß_{2}$-adrenoceptor stimulation with flow mediated dilatation. Br J Clin Pharmacol 2007;65:238-43.

[27] Holland DT, Godfredsen KA, Page T, Connor JD. Simple high-performance liquid chromatography method for the simultaneous determination of serum caffeine and paraxanthine following rapid sample preparation. J Chromatogr B Biomed Sci Appl 1998;707:105-10.

[28] Armstrong PC, Truss NJ, Ali FY, Dhanji AA, Vojnovic I, Zain ZN, et al. Aspirin and the in vitro linear relationship between thromboxane A2-mediated platelet aggregation and platelet production of thromboxane A2. J Thromb Haemost 2008;6:1933-43

[29] Gresele P, Momi S, Falcinelli E. Anti-platelet therapy: phosphodiesterase inhibitors. Br J Clin Pharmacol 2011;72:634-46.

[30] Prospective Studies Collaboration. Age-specific relevance of usual blood pressure to vascular mortality: a meta-analysis of individual data for one million adults in 61 prospective 
studies. Lancet 2002;360:1903-13.

[31] Akita M, Kuwahara M, Itoh F, Nakano Y, Osakabe N, Kurosawa T, et al. Effects of cacao liquor polyphenols on cardiovascular and autonomic nervous functions in hypercholesterolaemic rabbits. Basic Clin Pharmacol Toxicol 2008;103:581-7.

[32] Curtis BM, O'Keefe JH Jr. Autonomic tone as a cardiovascular risk factor: the dangers of chronic fight or flight. Mayo Clin Proc 2002;77:45-54.

[33] Custodis F, Schirmer SH, Baumhäkel M, Heusch G, Böhm M, Laufs U. Vascular pathophysiology in response to increased heart rate. J Am Coll Cardiol 2010;56:1973-83. [34] Avolio A, Van Bortel L, Boutouyrie P, Cockcroft JR, McEniery CM, Protogerou AD, et al. The role of pulse pressure amplification in arterial hypertension: experts' opinion and review of the Data. Hypertension 2009;54:375-83.

[35] Manisty CH, Hughes AD. Meta-analysis of the comparative effects of different classes of antihypertensive agents on brachial and central systolic blood pressure, and augmentation index. Br J Clin Pharmacol 2013;75:79-92.

[36] Williams B, Lacy PS; CAFE and the ASCOT (Anglo-Scandinavian Cardiac Outcomes Trial) Investigators. Impact of heart rate on central aortic pressures and hemodynamics: analysis from the CAFE (Conduit Artery Function Evaluation) study: CAFE-Heart Rate. J Am Coll Cardiol 2009;54:705-13.

[37] Sharman JE, McEniery CM, Dhakam ZR, Coombes JS, Wilkinson IB, Cockcroft JR. Pulse pressure amplification during exercise is significantly reduced with age and hypercholesterolemia. J Hypertens 2007;25:1249-54.

[38] Laurent P, Marenco P, Castagna O, Smulyan H, Blacher J, Safar ME. Differences in central systolic blood pressure and aortic stiffness between aerobically trained and sedentary individuals. J Am Soc Hypertens 2011;5:85-93.

[39] Curtis PJ, Potter J, Kroon PA, Wilson P, Dhatariya K, Sampson M, et al. Vascular 
function and atherosclerosis progression after $1 \mathrm{y}$ of flavonoid intake in statin-treated postmenopausal women with type 2 diabetes: a double-blind randomized controlled trial. Am J Clin Nutr 2013;97:936-42.

[40] Pereira T, Maldonado J, Laranjeiro M, Coutinho R, Cardoso E, Andrade I, et al. Central arterial hemodynamic effects of dark chocolate ingestion in young healthy people: a randomized and controlled trial. Cardiol Res Pract 2014;2014:945951.

[41] Lakatta EG, Levy D. Arterial and cardiac aging: major shareholders in cardiovascular disease enterprises: part I: aging arteries: a "set up" for vascular disease. Circulation 2003;107:139-46.

[42] Thorin E, Thorin-Trescases N. Vascular endothelial ageing, heartbeat after heartbeat. Cardiovasc Res 2009;84:24-32.

[43] Varughese GI, Lip GY Is hypertension a prothrombotic state? Curr Hypertens Rep $2005 ; 7: 168-73$.

[44] Smolenski A. Novel roles of cAMP/cGMP-dependent signaling in platelets. J Thromb Haemost 2012;10:167-76.

[45] Lopez-Garcia E, Rodriguez-Artalejo F, Rexrode KM, Logroscino G, Hu FB, van Dam RM. Coffee consumption and risk of stroke in women. Circulation 2009;119:1116-23. [46] Larsson SC, Virtamo J, Wolk A. Coffee consumption and risk of stroke in women. Stroke 2011;42:908-12.

[47] Kokubo Y, Iso H, Saito I, Yamagishi K, Yatsuya H, Ishihara J, et al. The impact of green tea and coffee consumption on the reduced risk of stroke incidence in Japanese population: the Japan public health center-based study cohort. Stroke 2013; 44:1369-74.

[48] Kris-Etherton PM, Derr J, Mitchell DC, Mustad VA, Russell ME, McDonnell ET, Salabsky D, Pearson TA. The role of fatty acid saturation on plasma lipids, lipoproteins, and apolipoproteins: I. Effects of whole food diets high in cocoa butter, olive oil, soybean oil, 
dairy butter, and milk chocolate on the plasma lipids of young men. Metabolism 1993;42:1219.

[49] Sever PS, Poulter NR, Elliott WJ, Jonsson MC, Black HR. Blood pressure reduction is not the only determinant of outcome. Circulation 2006;113:2754-74.

[50] Duprez DA. Systolic hypertension in the elderly: addressing an unmet need. Am J Med 2008;121:179-84.e3 
Table 1. Composition of high and low flavanol dark chocolate bars

Total cocoa solids (\%)

Flavanols:

monomers, $\mathrm{mg}$
procyanidin dimers, $\mathrm{mg}$
procyanidin trimers to decamers, $\mathrm{mg}$
total flavanols (monomers to decamers), $\mathrm{mg}$

Theobromine, mg

Caffeine, $\mathrm{mg}$

Total fat, $g$

saturated, $g$

monounsaturated, $g$

polyunsaturated, $g$

Total protein, $\mathrm{g}$

Available carbohydrates, $g$ sugars, $g$ starch, $g$

Dietary fibre, $g$

Calories, kcal

$\begin{array}{cc}\text { HFDC } & \text { LFDC } \\ 65 & 65 \\ & \\ 223 & 23 \\ 192 & 24 \\ 649 & 41 \\ 1064 & 88 \\ 395 & 420 \\ 35 & 35 \\ 19.9 & 18.8 \\ 12.4 & 11.7 \\ 6.9 & 6.6 \\ 0.6 & 0.5 \\ 2.8 & 2.9 \\ 18.2 & 18.8 \\ 15.8 & 16.4 \\ 2.4 & 2.4 \\ 3.0 & 3.1 \\ 264 & 257\end{array}$

Values are per $50 \mathrm{~g}$ bar (the daily amount consumed during the HFDC and LFDC phases of the study). 
Table 2 Characteristics of the study population at recruitment

Age (years)

Wt $(\mathrm{kg})$

BMI $\left(\mathrm{kg} / \mathrm{m}^{2}\right)$

Pre-/mild hypertension

SBP $(\mathrm{mmHg})$

$24 \mathrm{~h}$

day

night
$55.4 \pm 1.5$

$80.1 \pm 3.0$

$26.6 \pm 0.5$

$20 / 6$

$134.6 \pm 1.4$

$137.2 \pm 1.5$

$123.6 \pm 1.7$

$85.2 \pm 1.1$

$87.2 \pm 1.1$

$76.1 \pm 1.5$

$102.2 \pm 1.0$

$104.4 \pm 1.0$

$92.2 \pm 1.5$

$74.4 \pm 1.9$

$75.4 \pm 1.9$

$69.0 \pm 2.5$ 
Table 3 Comparison of $24 \mathrm{~h}$ ambulatory blood pressure and heart rate measurements after HFDC and LFDC compared to pre-study recordings $(n=21)$.

$\begin{array}{llll} & \text { Pre-Study } & \text { HFDC } & \text { LFDC } \\ \text { SBP }(\mathrm{mmHg}) & & & \\ 24-\mathrm{h} & 135.2 \pm 1.7 & 133.1 \pm 1.9 & 134.1 \pm 1.9 \\ \text { day } & 137.6 \pm 1.6 & 135.4 \pm 2.0 & 136.8 \pm 1.9 \\ \text { night } & 124.2 \pm 2.0 & 124.1 \pm 2.7 & 122.2 \pm 2.2\end{array}$

DBP (mmHg)

$\begin{array}{llll}24-h & 85.1 \pm 1.3 & 84.0 \pm 2.0 & 84.9 \pm 1.4 \\ \text { day } & 87.1 \pm 1.3 & 85.7 \pm 2.0 & 87.0 \pm 1.4 \\ \text { night } & 76.3 \pm 1.7 & 76.9 \pm 2.2 & 75.0 \pm 1.7\end{array}$

MAP (mmHg)

$\begin{array}{lrrr}\text { 24-h } & 102.3 \pm 1.3 & 100.5 \pm 1.9 & 101.7 \pm 1.4 \\ \text { day } & 104.4 \pm 1.2 & 102.3 \pm 1.9 & 103.9 \pm 1.4 \\ \text { night } & 92.3 \pm 1.7 & 93.0 \pm 2.3 & 90.9 \pm 1.8\end{array}$

HR (bpm)

$\begin{array}{lllll}\text { 24-h } & 72.9 \pm 2.2 & 73.1 \pm 2.1^{\mathrm{a}} & 77.2 \pm 2.3^{\mathrm{b}} & \text { ANOVA P }=0.004 \\ \text { day } & 73.8 \pm 2.0 & 75.1 \pm 2.1^{\mathrm{c}} & 79.2 \pm 2.1^{\mathrm{d}} & \text { ANOVA P }=0.001 \\ \text { night } & 67.5 \pm 2.9 & 68.5 \pm 2.8 & 69.7 \pm 3.2 & \end{array}$

Mean data from 21 subjects with ambulatory blood pressure measurements at all three time points.

${ }^{\mathrm{a}} \mathrm{P}=0.005$ vs LFDC, ${ }^{\mathrm{b}} \mathrm{P}=0.008$ vs pre-study, ${ }^{\mathrm{c}} \mathrm{P}=0.002$ vs LFDC, ${ }^{\mathrm{d}} \mathrm{P}=0.001$ vs pre-study. 
Table 4 Basal brachial artery (peripheral) measurements and aortic (central) values derived from pulse wave analyses after 6 weeks of HFDC and LFDC prior to salbutamol administration $(\mathrm{n}=26)$.

$\begin{array}{lll} & \text { HFDC } & \text { LFDC } \\ \text { Peripheral systolic blood pressure (PSBP, mmHg) } & 132.5 \pm 2.0 & 133.4 \pm 1.6 \\ \text { Peripheral diastolic blood pressure (PDBP, mmHg) } & 81.3 \pm 1.3 & 80.9 \pm 1.3 \\ \text { Peripheral pulse pressure (PPP, mmHg) } & 51.2 \pm 1.5 & 52.5 \pm 1.6 \\ \text { Peripheral mean artery blood pressure (PMAP, mmHg) } & 99.5 \pm 1.6 & 99.7 \pm 1.3 \\ \text { Central systolic blood pressure (CSBP, mmHg) } & 121.9 \pm 2.1 & 121.5 \pm 1.5 \\ \text { Central diastolic blood pressure (CDBP, mmHg) } & 82.4 \pm 1.4 & 82.4 \pm 1.3 \\ \text { Central pulse pressure (CPP, mmHg) } & 39.5 \pm 1.6 & 39.1 \pm 1.3 \\ \text { Heart Rate (HR, bpm) } & 67.3 \pm 2.2^{\mathrm{a}} & 71.0 \pm 2.4 \\ \text { Augmentation Pressure (AP, mmHg) } & 10.3 \pm 1.1 & 9.4 \pm 0.9 \\ \text { Augmentation Index (AI, \%) } & 20.7 \pm 2.1 & 21.5 \pm 1.8 \\ \text { PSBP - CSBP (mmHg) } & 10.6 \pm 0.6^{\mathrm{b}} & 11.9 \pm 0.9 \\ \text { Pulse pressure amplification ratio (PPA) } & 1.31 \pm 0.03^{\mathrm{c}} & 1.36 \pm 0.03\end{array}$

Paired t-test comparison of basal values HFDC v LFDC: ${ }^{\mathrm{a}} \mathrm{P}=0.022 ;{ }^{\mathrm{b}} \mathrm{P}=0.029 ;{ }^{\mathrm{c}} \mathrm{P}=0.031$ 
Table 5 Comparison of methylxanthine levels and lipid profiles at baseline with average values after HFDC and LFDC. Methylxanthine measurements were made on plasma samples collected after 6 weeks consumption of the respective products. Lipid values for HFDC and LDFC are the means of values obtained after 3 and 6 weeks consumption of each product

$\begin{array}{llll} & \text { Baseline } & \text { HFDC } & \text { LFDC } \\ \text { Theobromine }(\mu \mathrm{g} / \mathrm{ml}) & 0.77 \pm 0.14 & 4.49 \pm 0.48^{\mathrm{a}} & 4.34 \pm 0.51^{\mathrm{a}} \\ \text { Caffeine }(\mu \mathrm{g} / \mathrm{ml}) & 1.24 \pm 0.19 & 1.31 \pm 0.20 & 1.28 \pm 0.19 \\ \text { Cholesterol }(\mathrm{mmol} / \mathrm{L}) & 5.71 \pm 0.19 & 5.60 \pm 0.14 & 5.70 \pm 0.16 \\ \text { LDL-C }(\mathrm{mmol} / \mathrm{L}) & 3.53 \pm 0.15 & 3.44 \pm 0.14 & 3.48 \pm 0.13 \\ \text { HDL-C }(\mathrm{mmol} / \mathrm{L}) & 1.57 \pm 0.06 & 1.56 \pm 0.05 & 1.57 \pm 0.06 \\ \text { Triglycerides }(\mathrm{mmol} / \mathrm{L}) & 1.36 \pm 0.10 & 1.39 \pm 0.11 & 1.45 \pm 0.11 \\ & & & \\ & & & \\ & & & \end{array}$




\section{FIGURES}
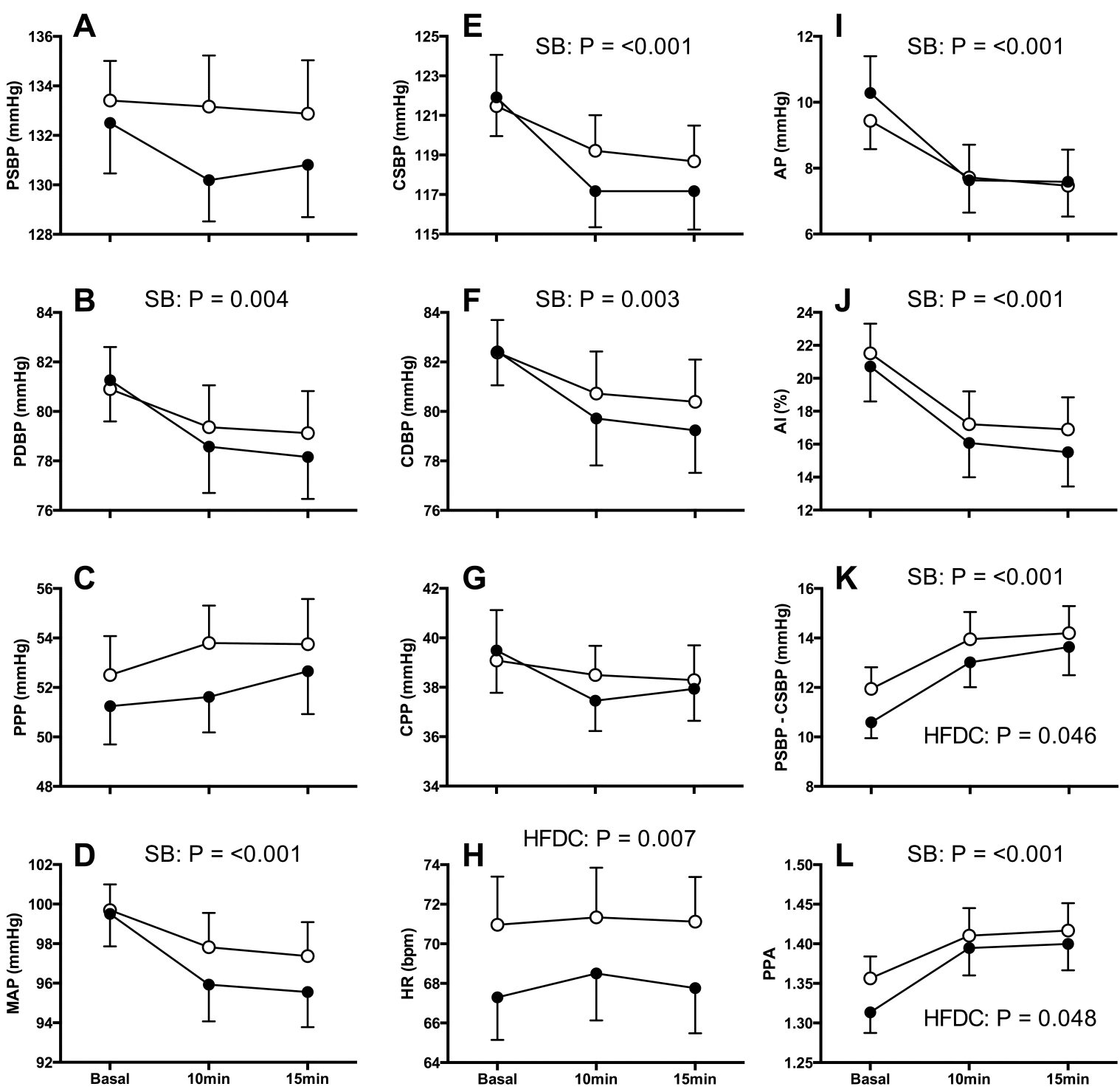

Figure 1 Effect of 6 weeks daily consumption of HFDC and LFDC on responses to salbutamol determined by pulse wave analysis (abbreviations as Table 4). P values: comparison of HFDC (solid circles) v LFDC (open circles); and comparison of salbutamol responses v basal values. 

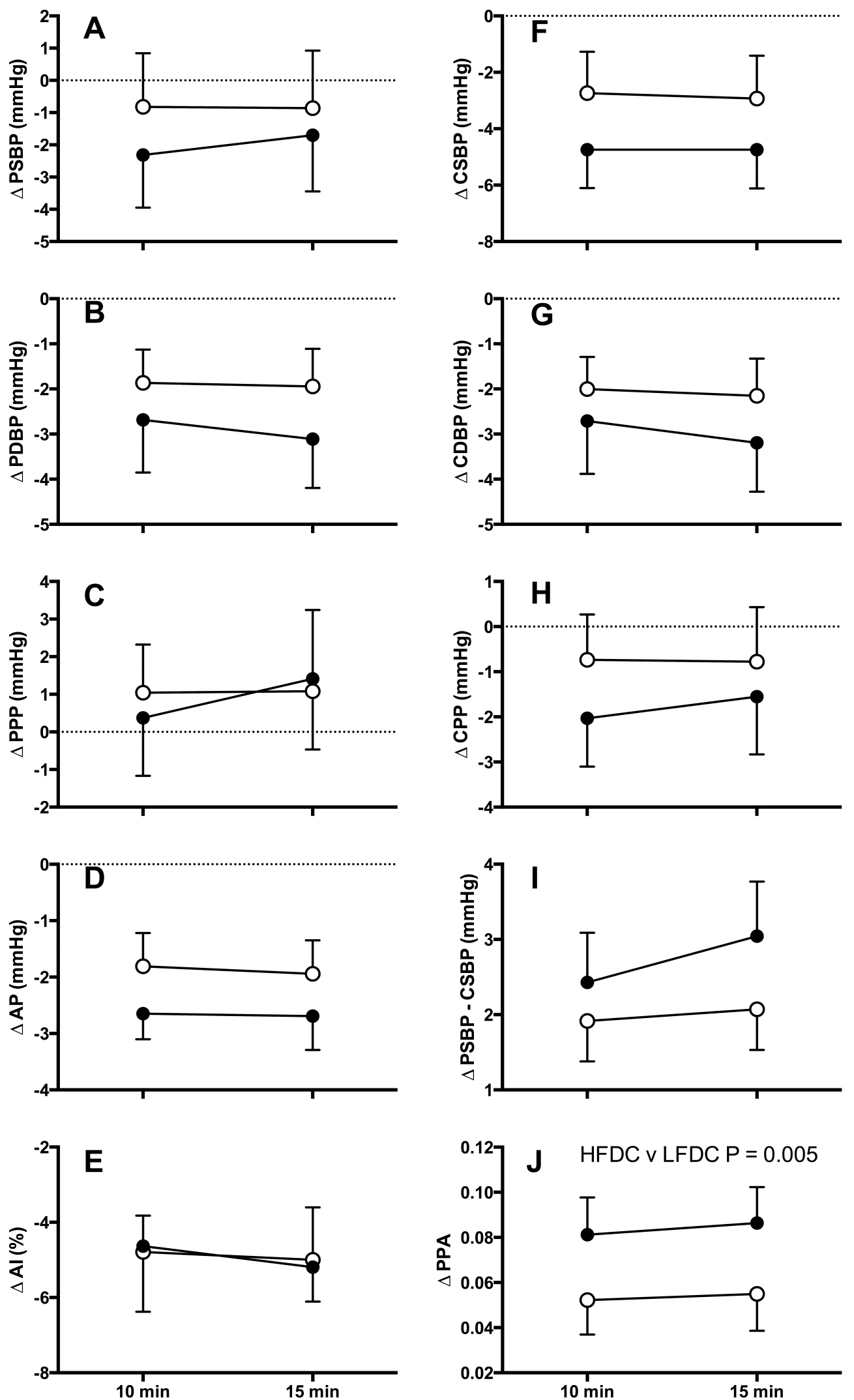

Figure 2 Mean changes from basal after salbutamol inhalation for parameters determined during pulse wave analyses, HFDC (solid circles) and LFDC (open circles). 

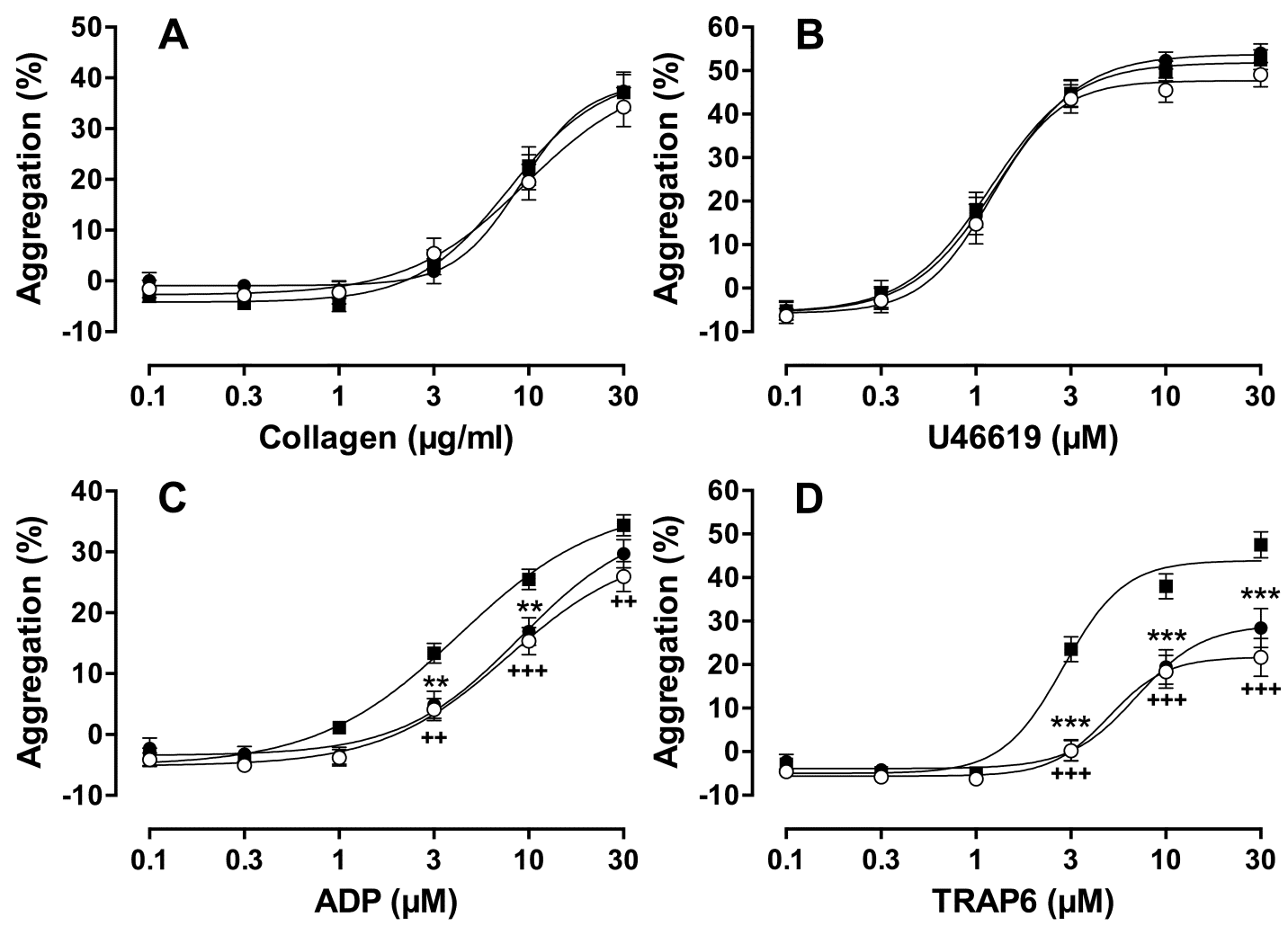

Figure 3 Comparative concentration response curves for platelet aggregation stimulated with (a) collagen, (b) U46619, (c) ADP and (d) TRAP6. Baseline responses prior to chocolate consumption (solid squares) compared with those after 6 weeks HFDC (solid circles) and 6 weeks LFDC (open circles). Data are from 22 subjects studied at all time points; $* *,{ }^{++} \mathrm{P}=<0.01$; and $* * *,{ }^{+++} \mathrm{P}=<0.001$ versus baseline responses. 
A
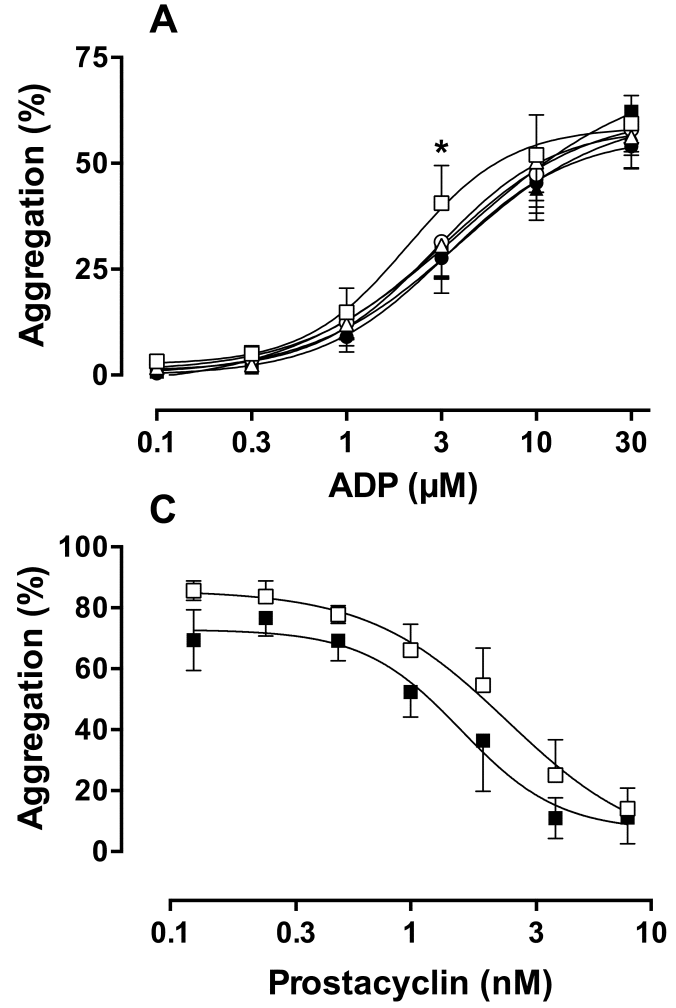

B
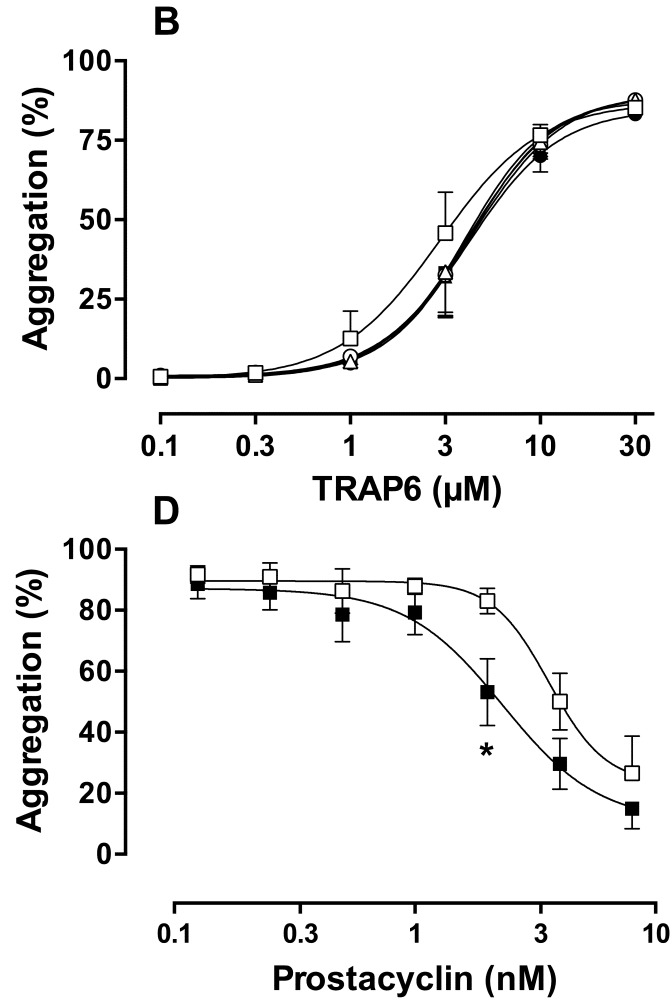

Figure 4 Effect of theobromine on platelet aggregation induced by (a) ADP and (b) TRAP6 (open squares vehicle control; theobromine: $2 \mu \mathrm{g} / \mathrm{ml}$ open triangles, $5 \mu \mathrm{g} / \mathrm{ml}$ open circles, $10 \mu \mathrm{g} / \mathrm{ml}$ solid triangles, $20 \mu \mathrm{g} / \mathrm{ml}$ solid squares, $50 \mu \mathrm{g} / \mathrm{ml}$ solid circles; $\mathrm{n}=5$ for each). Effect of prostacyclin on platelet aggregation induced with (c) $10 \mu \mathrm{M}$ ADP, or (d) $10 \mu \mathrm{M}$ TRAP6, either alone (open squares) or in the presence of $20 \mu \mathrm{g} / \mathrm{ml}$ theobromine (solid squares). $* \mathrm{P}=<0.05$ versus response without theobromine. 